\title{
Elaboración de una galleta a base de harinas de plátano pelipita (Musa abb) y de batata (Ipomea batatas)
}

\section{Preparation of a biscuit based on banana (Musa abb) and sweet potato (Ipomea batatas) flours}

\begin{abstract}
RESUMEN
Con la finalidad de diversificar el uso de las harinas de plátano pelipita (Musa ABB) y batata (Ipomoea batatas), se propuso evaluar la funcionalidad de una galleta sustituyendo la harina de trigo con $70 \%$ de harina de plátano pelipita y $30 \%$ harina de batata con el fin de obtener un producto con propiedades físicas y organolépticas agradables, además de mejorar la calidad nutricional, en cuanto a fibra dietaría y almidones resistentes. Las galletas elaboradas a base de harina de plátano pelipita y batata incrementaron significativamente, con respecto a la galleta patrón (GP), en los siguientes componentes químicos: ceniza (0,6 a 2,1\%), proteínas (de 3,9 a 5,3\%), fibra dietaría (4,1 a 5,3\%) y azucares totales $(24,1$ a 22,1\%). La galleta de harina de plátano y batata cumple con los requerimientos establecidos en la industria en cuanto a humedad $(3,3 \%)$, aw $(0,410)$ y color ( $L=49,3 a=4,79$ y $b=19,3)$. Los resultados mostraron que la harina compuesta, contribuyó a un ligero incremento en las fracciones de fibra, proteínas, ceniza, fósforo, calcio y hierro en las galletas. En conclusión, el uso de la harina de batata y plátano en una relación de $30 \%$ - 70\% respectivamente, resultó un ingrediente adecuado en la elaboración de galletas con alta preferencia sensorial, constituyendo una alternativa como fuente de fibra dietética.

Palabras clave: Galletas; Harinas compuestas.
\end{abstract}

\begin{abstract}
In order to diversify the use of Pelipita banana (Musa ABB) and sweet potato (Ipomoea batatas) flours, we proposed evaluating the functionality of a cookie replacing wheat flour with $70 \%$ Pelipita banana flour and 30\% sweet potato flour in order to obtain a product with pleasant organoleptic and physical properties and improved nutritional quality, in terms of dietary fiber and resistant starch. Cookies prepared from Pelipita banana and sweet potato flours increased significantly with respect to the following chemical components: ash (from 0.6 to $2.1 \%$ ), protein (3.9 to $5.3 \%$ ), dietary fiber (4.1 to $5.3 \%$ ) and total sugars (24.1 to $22.1 \%$ ). Cookies made with banana and sweet potato flour met the requirements established in the industry in terms of moisture (3.3\%), aw (0.410) and color $(L=49.3$
\end{abstract}

Glicerio Leon-Mendez ${ }^{1 *}$, Deisy Leon-Mendez ${ }^{1}$, Nerlis Pajaro-Castro ${ }^{2}$, Clemente Granados-Conde ${ }^{3}$, Edgard Granados-Llamas³, María José Bahoque Peña ${ }^{4}$.

1. Facultad de Ciencias de la Salud. Corporación Universitaria Rafael Núñez. Programa de Tecnología en Estética y Cosmetología. GITEC, Cartagena, Colombia.

2. Facultad de Ciencias de la Salud, Universidad de Sucre y Grupo de Ciencias Médicas y Farmacéuticas, Sucre, Colombia. 3. Facultad de Ingeniería, Universidad de Cartagena y Grupo de Investigación, Ingeniería, Innovación, Calidad Alimentaria y Salud (INCAS). Universidad de Cartagena, Cartagena, Colombia.

4. Facultad de Ciencias Farmacéuticas, Universidad de Cartagena. Grupo de investigación en Tecnología Farmacéutica, Cosmética y de Alimentos (GITFCA). Universidad de Cartagena, Cartagena, Colombia.

*Dirigir correspondencia a: Glicerio Leon-Mendez. Facultad de Ciencias de la Salud. Corporación Universitaria Rafael Núñez. Programa de Tecnología en Estética y Cosmetología. GITEC, Cartagena, Colombia. E-mail: gleonm22@gmail.com

Este trabajo fue recibido el 16 de abril de 2019. Aceptado con modificaciones: 16 de diciembre de 2019 . Aceptado para ser publicado: 26 de febrero de 2020.

$a=4.79$ and $b=19.3)$. The results showed that the composite flour, contributed to a slight increase in fractions of fiber, protein, ash, phosphorus, calcium and iron in cookies. In conclusion, the use of sweet potato and banana flours in a ratio of $30 \%-70 \%$ respectively, was a suitable ingredient in the production of biscuits with high sensory preference, constituting an alternative source of dietary fiber.

Key words: Composite flours; Cookies.

\section{INTRODUCCION}

Colombia es un país que se caracteriza por tener una posición geográfica privilegiada, cerca de la línea del Ecuador, que lo hace potencialmente rico en fauna y flora'. Por lo mismo, presenta un clima favorable que permite que 
las regiones que lo conforman sean parte del crecimiento y del desarrollo rural del país debido a la calidad de los productos agrícolas que producen².

El plátano es una planta herbácea monocotiledónea, de la familia Musaceae, originaria del sudeste asiático y traída a nuestro país por los españoles en el siglo $\mathrm{XVI}^{3}$. Es considerado el cuarto cultivo más importante del mundo, por tratarse de un producto básico y de exportación, fuente de empleo e ingresos en numerosos países del trópico y subtrópico. Las variedades de plátano cultivadas en Colombia son: dominico-hartón, dominico, hartón, pelipita, morado, cachaco, popocho, pompo, maqueño, guineo y trucho ${ }^{4}$.

En Colombia la batata y plátano variedad pelipita se cultivan únicamente en huertas y se consume a nivel casero. También, son utilizadas en algunos casos para alimentación animal. Esto ocurre porque no se conocen los aportes nutricionales al ser humano, ni las ventajas de propagación vegetativa y el alto rendimiento agronómico que posee ${ }^{5}$. Además, la batata es una materia prima fundamental para el desarrollo de productos industriales innovadores, gracias a sus características sensoriales como sabor, color y tamaño $0^{6,7}$. En Colombia, la batata y el plátano variedad pelipita se pueden adquirir con módicos valores, sin embargo, para que estos alimentos sean consumidos habitualmente deben estar en una matriz con cualidades organolépticas aptas y de fácil consumo. Por lo tanto, el objetivo de este trabajo de investigación fue caracterizar bromatológicamente las harinas plátano pelipita (Musa $A B B)$ y de batata (Ipomea batatas) y desarrollar una galleta, como una alternativa nueva alimentaria para la población regional y nacional.

\section{MATERIALES Y MÉTODOS}

El plátano pelipita (Musa ABB) se compró en el municipio del Carmen de Bolívar, ubicado en el departamento de Bolívar $\left(9^{\circ} 43^{\prime} 06^{\prime \prime} \mathrm{N} 75^{\circ} 07^{\prime} 21^{\prime \prime} \mathrm{O}\right)$ y la batata (Ipomea batatas) en el municipio de Corozal, ubicado en el departamento de Sucre $\left(9^{\circ} 19^{\prime} 01^{\prime \prime} \mathrm{N} 75^{\circ} 17^{\prime} 36^{\prime \prime} \mathrm{O}\right)$. Las especies vegetales fueron recolectadas en madurez de consumo, y fueron seleccionados frescos, enteros y sin ningún tipo de deterioro mecánico ni microbiológico (Figura 1).

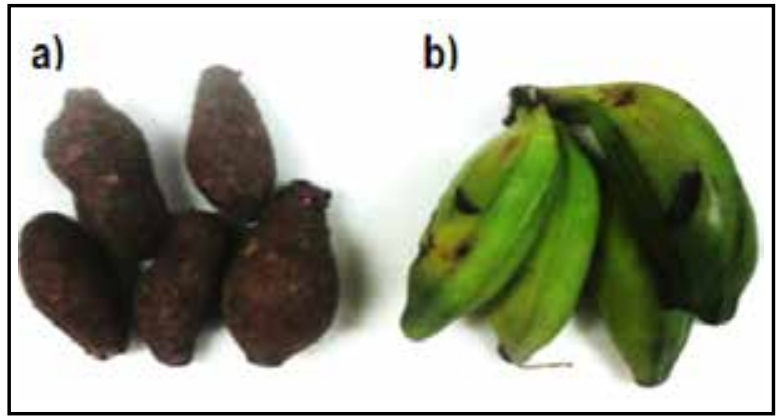

Figura 1: a) Batata (Ipomea batatas); b) Plátano pelipita (Musa ABB).

\section{Obtención harinas de plátano variedad pelipita (Musa ABB) y de batata (Ipomea batatas)}

Inicialmente, se escogió el material fresco previamente seleccionado y se le realizó el proceso de limpieza con agua corriente para retirar polvo, tierra y suciedad en general, posteriormente se desinfectó con Citrosan al 2.5\%. Luego se procedió al descortezado, seguidamente fueron cortada en rodajas con espesor de $2 \mathrm{~mm}$ y llevadas a un proceso de secado por flujo de aire caliente, a $40{ }^{\circ} \mathrm{C}$ por $6 \mathrm{~h}$ con velocidad de aire de $5 \mathrm{~m} / \mathrm{s}$, hasta alcanzar un contenido constante de humedad entre 10 a $12 \%$. Una vez secadas, fueron pasadas por un molino de martillos y un proceso de tamizaje con tamices $80-100$ durante 5 min $^{8,9}$.

\section{Caracterización bromatológica de las harinas obtenidas especies vegetales}

Se llevó a cabo la caracterización química de las harinas, a la cual se le determinó el contenido de nutrientes y micronutrientes mediante las pruebas descritas a continuación: proteína; se utilizó el método de Kjeldahl según AOAC 955.04; cenizas; mediante el método de directo según AOAC 924.05; humedad; por medio del método de secado a $100+2{ }^{\circ} \mathrm{C}$ según AOAC 925.0910; fibra ${ }^{11}$; por el método enzimático gravimétrico; carbohidratos ${ }^{8}$; grasa; por el método de Soxhlet según AOAC 936.15 ${ }^{12}$, minerales mediante la técnica instrumental de espectrofotometría de absorción atómica ${ }^{13,14}$.

\section{Elaboración de galletas}

La fórmula para la elaboración de las galletas se tomó de la Norma Técnica Colombiana (NTC-1241) para la elaboración de galletas. El procedimiento de elaboración de la galleta a base de harinas de plátano pelipita (HP) y batata (HB), consistió en someter a los ingredientes seleccionados a un proceso de mezclado, filtrado, secado a una temperatura de $180^{\circ} \mathrm{C}$, laminado y enrollado de la masa seca, posteriormente se procedió a cortado, enfriamiento y empaque, como ingredientes primarios se utilizaron harinas compuestas a partir de una mezcla de las harinas de plátano y de batata en una relación de HP:HB de 70:30\%.

\section{Mediciones instrumentales}

Para determinar el color de las galletas se realizaron cuatro mediciones con un colorímetro triestímulo Hunter Lab, registrándose los valores $L^{*}$ [luminosidad, de 0 (oscuro) a 100 (claro)], a* [de - 60 (verde) a + 60 (rojo)], b* [de 60 (azul) a +60 (amarillo)]. La textura de las galletas se determinó en un Texturómetro universal. Registrando la fuerza máxima de corte en Newtons ${ }^{6}$.

\section{Análisis sensorial}

Para evaluar el grado de aceptación y los atributos sensoriales de la formulación se utilizó la escala Hedónica de 6 puntos: aceptabilidad, donde 1 (me disgusta mucho), 2 (me disgusta), 3 (ni me gusta ni me disgusta), 4 (me gusta ligeramente), 5 (me gusta), 6 (me gusta mucho) por un panel 
de expertos conformado por 50 panelistas. Los panelistas registraron el grado de gusto o disgusto en términos que mejor describen su percepción sobre el producto ${ }^{13}$.

\section{Análisis estadístico}

Los ensayos se realizaron por triplicado con el fin de garantizar resultados analíticos confiables mediante el programa de GraphPad Prism 5. La distribución normal y la igualdad entre la varianza se verificaron mediante las pruebas de Kolmogorov-Smirnov y Bartlett, respectivamente. Los resultados se expresaron en media \pm EEM (error estándar de la media). Las diferencias significativas se determinaron mediante análisis de t de Student con valores de $p<0,05$ como diferencias significativas.

\section{RESULTADOS}

En la tabla 1 se reportan los valores de la composición proximal de las harinas. Se identificó que las harinas de batata y plátano variedad pelipita poseen un mayor aporte de minerales como calcio, fósforo y hierro, encontrándose diferencias significativas $(p<0,050)$ en los valores.

En la tabla 2 se presentan los resultados de la composición proximal de las galletas de harina de trigo (control) y de harina de batata y plátano en proporciones (30:70). Es importante señalar que se evidencian diferencias significativas $(p>0,050)$ en diferentes parámetros proximales de las galletas de (HP: HB de 70:30\%) con respecto al control.

Al medir instrumentalmente el color de las galletas se encontraron variaciones entre las formulaciones.

En la tabla 2 se muestran los resultados de los parámetros medidos (a*, b* y L). Se encontraron diferencias significativas $(p<0,050)$ en los valores de $a^{*}, b^{*}$ y $L$ para la formulación (HP:HB de 70:30\%).

Se evidencia en los resultados correspondiente al análisis sensorial de las diferentes de galletas, que existe un alto porcentaje de aceptación de los atributos sensoriales de la formulación (HP:HB de 70:30\%) con un (72 $\pm 0,55 \%$ ) en comparación con las galletas de trigo que presentaron un resultado del $(28 \pm 0,25 \%)$. Identificando la formulación (HP:HB de $70: 30 \%$ ) como producto de calidad, la cual posee sabores persistentes acordes con una galleta con fibra, crujientes y dulces.

\section{DISCUSIÓN}

En general, las galletas son productos elaborados básicamente con harinas de trigo, avena o centeno, azúcar y grasa vegetal, cuyo contenido proteico mínimo debe ser de cinco a ocho $\%^{6}$. Los valores proteicos $(5,25 \pm 0,22)$ de las galletas HP:HB de 70:30\% cumplen dicha especificación química y son menores que los resultados obtenidos en galletas con distintos tipos de harinas. Román \& Valencia ${ }^{15}$ reportaron un contenido proteico de $8,1 \%$ para galletas elaboradas con fibras de cereales, Granito et al. ${ }^{16}$ reportaron $10,1 \%$ de proteínas en galletas elaboradas con $30 \%$ de frijol; Escobar et al..$^{17}$ reportaron 10,7-13,3\% de proteínas en galletas hechas con harina de cotiledón de algarrobo; Ceron et al. ${ }^{9}$ reportaron un valor de proteínas comprendido entre 9,6-12,6\% para galletas a base de harina de papa de la variedad Parda Pastusa (Solanum tuberosum L.); DelgadoVidal et al. ${ }^{6}$ indicaron los valores proteicos $(8,8-13,9 \%)$ de las galletas enriquecidas con carne de barrilete. Aunque, la galleta elaborada contiene valores proteicos inferiores a los reportes mencionados, es importante destacar la calidad

Tabla 1. Resultados de la composición proximal de las harinas.

\begin{tabular}{|lccc|}
\hline Parámetros & Harina de trigo (control) & Harina de batata & Harina de plátano \\
\hline Actividad de agua (aW) & $0,95 \pm 0,02^{*}$ & $0,82 \pm 0,03^{*}$ & $0,79 \pm 0,02^{*}$ \\
Humedad (\%) & $9,57 \pm 0,40^{*}$ & $7,21 \pm 0,19^{*}$ & $12,8 \pm 0,76^{*}$ \\
Ceniza (\%) & $0,930 \pm 0,04^{*}$ & $3,92 \pm 0,03^{*}$ & $2,96 \pm 0,16^{*}$ \\
Proteína (\%) & $8,87 \pm 0,15^{*}$ & $10,0 \pm 0,05^{*}$ & $5,17 \pm 0,29^{*}$ \\
Grasas (\%) & $0,870 \pm 0,03^{*}$ & $0,850 \pm 0,05^{*}$ & $0,350 \pm 0,02^{*}$ \\
Carbohidratos (\%) & $79,8 \pm 0,62^{*}$ & $77,5 \pm 0,31^{*}$ & $78,7 \pm 0,85^{*}$ \\
Fibra dietaría (\%) & $2,33 \pm 0,12^{*}$ & $4,47 \pm 0,15^{*}$ & $3,03 \pm 0,06^{*}$ \\
Azucares reductores & $0,500 \pm 0,05^{*}$ & $0,630 \pm 0,03^{*}$ & $0,610 \pm 0,02^{*}$ \\
P (mg) & $91,3 \pm 3,21^{*}$ & $103 \pm 2,08^{*}$ & $107 \pm 2,61^{*}$ \\
Ca (mg) & $29,7 \pm 2,52^{*}$ & $147 \pm 2,58^{*}$ & $31,17 \pm 0,76^{*}$ \\
Fe (mg) & $4,67 \pm 0,29^{*}$ & $6,20 \pm 0,20^{*}$ & $4,37 \pm 0,20^{*}$ \\
& & & \\
\hline
\end{tabular}

* Diferencias significativas comparado con la harina de trigo $100 \%$, $\mathrm{p}<0.05$. 
Tabla 2. Resultados de la composición proximal de las galletas.

\begin{tabular}{|lcc|}
\hline Parámetros & Galleta PyB & Galleta de trigo (100\%) \\
\hline Actividad de agua (aW) & $0,410 \pm 0,01^{*}$ & $0,430 \pm 0,02$ \\
Humedad (\%) & $3,35 \pm 0,05^{*}$ & $3,83 \pm 0,15$ \\
Ceniza (\%) & $2,10 \pm 0,05^{*}$ & $0,620 \pm 0,04$ \\
Proteína (\%) & $5,25 \pm 0,22^{*}$ & $3,93 \pm 0,12$ \\
Grasas (\%) & $0,510 \pm 0,04^{*}$ & $1,20 \pm 0,26$ \\
Carbohidratos (\%) & $88,8 \pm 0,31^{*}$ & $91,4 \pm 0,27$ \\
Fibra dietaría (\%) & $5,23 \pm 0,23^{*}$ & $4,08 \pm 0,10$ \\
Azucares totales & $22,1 \pm 0,32^{*}$ & $24,1 \pm 0,32$ \\
Azucares reductores & $3,03 \pm 0,06^{*}$ & $2,30 \pm 0,26$ \\
P (mg) & $74,0 \pm 3,61^{*}$ & $2,87 \pm 0,12$ \\
Ca (mg) & $19,7 \pm 1,15^{*}$ & $0,360 \pm 0,01$ \\
Fe (mg) & $3,05 \pm 0,05^{*}$ & $2,67 \pm 0,15$ \\
L* & $49,0 \pm 0,84^{*}$ & $51,6 \pm 0,55$ \\
a* & $4,79 \pm 0,02^{*}$ & $5,82 \pm 0,025$ \\
b* & $19,3 \pm 0,25^{*}$ & $21,3 \pm 0,25$ \\
Calorías (Kilocalorías/100g) & $385 \pm 0,35^{*}$ & $388 \pm 1,65$ \\
Textura (N) & $68,83 \pm 1,04^{*}$ & $54,2 \pm 0,76$ \\
& & \\
\hline
\end{tabular}

Galleta PyB: Galleta con harina de plátano y batata (70:30).

L: valores promedio de Luminosidad, a: valores promedio del matiz (+ rojo, - verde), b: valores promedio de la tonalidad (+ amarillo, - azul).

* Diferencias significativas comparado con la galleta de trigo 100\%, p<0.05.

del aporte proteico de la harina de Ipomea batatas, el cual es muy valioso dado que contiene aminoácidos esenciales; tales como: leucina $(0,092 \mathrm{~g} / 100 \mathrm{~g})$, isoleucina $(0,055 \mathrm{~g} / 100 \mathrm{~g})$, lisina $(0,066 \mathrm{~g} / 100 \mathrm{~g})$, metionina $(0,029 \mathrm{~g} / 100 \mathrm{~g})$, fenilalanina $(0,089 \mathrm{~g} / 100 \mathrm{~g})$, treonina $(0,083 \mathrm{~g} / 100 \mathrm{~g})$, triptófano $(0,031$ $\mathrm{g} / 100 \mathrm{~g})$, valina $(0,086 \mathrm{~g} / 100 \mathrm{~g})$ e histidina $(0,031 \mathrm{~g} / 100 \mathrm{~g})^{18}$.

La $a_{\omega}$ es una de las propiedades más importantes de los alimentos deshidratados ya que determina en gran medida los cambios químicos, bioquímicos, microbiológicos y físicos que se pueden presentar durante su almacenamiento y que alteran su vida útil ${ }^{6}$. En este sentido, los valores bajos de $a \omega(0,410 \pm 0,010)$ para las galletas formuladas en esta investigación, se deben a que típicamente los azúcares enlazan el agua en el sistema alimentario ${ }^{6}$.

El color característico de las formulaciones está asociado al efecto de la temperatura y tiempo de horneado sobre el contenido de azúcar, lípidos, proteínas y almidón, los cuales al parecer originaron una coloración típica de las reacciones de Maillard. En un producto cuanto mayor sea la cantidad de azúcares presentes, incluyendo los no reductores se desarrollará un color marrón más oscuro en su superficie ${ }^{6}$.

La fibra es un componente presente en el plátano variedad pelipita la cual es difícilmente degradable debido a sus componentes como la lignina, celulosa y hemicelulosa ${ }^{3}$. Lo cual se evidencia con valores $(5,23 \pm 0,25)$ de las galletas HP:HB de 70:30\% y en el análisis sensorial fueron evaluadas como producto de calidad alta-media; tienen sabores persistentes acordes con una galleta con fibra, especialmente a harina, dulce y salvado ${ }^{15}$.

\section{CONCLUSIONES}

Se puede concluir que la mezcla de un $70 \%$ de harina de plátano pelipita y $30 \%$ de batata en la elaboración de galletas aumenta su contenido de proteínas, respecto a trigo, así como aumenta el contenido de fibra dietética, sin afectar sus características físicas ni la aceptabilidad sensorial evaluada para el producto.

Agradecimientos. Los autores agradecen a las Corporación Universitaria Rafael Núñez, Universidades de Cartagena, Sucre por facilitar espacio, recursos y tiempo de los investigadores.

\section{BIBLIOGRAFÍA}

1. Granados-Conde C, Torrenegra-Alarcon M, Tejada-Tovar C. Characterization of the pulp of Physalis peruviana L. cultivated in the department of north of Santander-Colombia. BISTUA. 
2017; 15: 1-10.

2. Tejada-Tovar C, Granados-Conde C, Villabona-Ortiz A. Characterization pulp of tabasco peppers (Capsicum frutescens L.) cultivated in the department of Sucre-Colombia. BISTUA. 2017; 15: 11-20.

3. Granados C, Acevedo D, Cabeza A, Lozano A. Texture profile analysis in bananas Pelipita, Hartón and Topocho. Inf Tecnol. 2014; 25: 35-40.

4. Corporación Colombiana de Investigación Agropecuaria, Corpoica. Sustainable Management of banana cultivation [Internet]. 2006. [Consultado 3 Dic 2019]. Disponible en: http://www.corpoica.org.co/sitioweb/Archivos/Publicaciones/ Cultivodelpltano.pdf

5. Blasco-López G, Gómez-Montaño FJ. Functional properties of banana (Musa sp). Rev Med UV. 2014; 2: 22-26.

6. Delgado-Vidal FK, Ramirez-Rivera E, Rodriguez-Miranda J, Martinez-Lopez RE. Preparation of cookies enriched with black skipjack (Euthynnus lineatus): chemical, instrumental and sensory characterisation. Universidad \& Ciencia 2013; 29: 287-300.

7. López-Villafuerte $K$, Cabrera-Martínez D, Águilar-Nájera $O$, Sol-González W, López-Zúñiga E, Vela-Gutiérrez G. Evaluation of the nutritional impact and the organoleptic aceptance of enriched cookies with whey, soy and macadamia nuts in preschool community of Chiapas, Mexico. Ciencia UAT. 2013; 8: 33-41.

8. Rodriguez-Sandoval E, Lascano A, Sandoval G. Influence of the partial substitution of wheat flour for quinoa and potato flour on the thermomechanical and breadmaking properties of dough. Rev UDCA Actual. Divulg Cient. 2012; 15: 199207.

9. Cerón-Cardenas A, Bucheli-Jurado M, Osorio-Mora O. Development of biscuit made from potato flour variety Parda Pastusa (Solanum tuberosum L.). Acta Agron. 2014; 63: 101-109.

10. AOAC. Official methods of analysis of the association of official analytical chemist 16th Ed. Association of official analytical chemists. Washington DC, USA [Internet]. 1995 [Consultado 3 Dic 2019]. Disponible en: https://www.worldcat. org/title/official-methods-of-analysis-of-aoac-international/ oclc/421897987.

11. Torrenegra M, Villalobos O, Castellar E, León G, Granados C, Pajaro N. Evaluation of the antioxidant activity of pulps from Rubus glaucus B., Vaccinium floribundum K. and Beta vulgaris L. Rev Cubana Plant Med. 2016; 21: 1-8.

12. AOAC. Official methods of analysis of the association of official analytical chemist, 15th ed. Methods 932.06, 925.09, 985.29, 923.03. Association of official analytical chemists. Arlington, VA, USA [Internet]. 1990 [Consultado 15 Dic 2019]. Disponible en: https://www.worldcat.org/title/official-methodsof-analysis-of-the-association-of-official-analytical-chemists/ oclc/20709424

13. Pajaro N, Torrenegra M, Granados C, Leon G, Pajaro E, Osorio M. Microencapsulation of pulp of Mangifera indica L. by spray drying and antioxidant activity. Int I Pharm Pharm Sci. 2017; 9: 181-185.

14. León G, Granados C, Osorio M. Characterization of the pulp of Annona Muricata L. Cultivated in the North of the Bolivar Department - Colombia. Rev Cubana Plant Med. 2016; 21: 1-9.

15. Román M, Valencia FE. Evaluation of crackers with cereal fiber as a functional food. VITAE (Medellin). 2006; 13: 36-43.

16. Granito $M$, Valero $Y, Z$ Zambrano R. Baked product development based fermented legumes and cereals for schoolchildren snack. Arch Latinoam Nutr. 2010; 60: 85-92.

17. Escobar B, Estévez AM, Fuentes GC, Venegas FD. Use of algarrobo (Prosopis chilensis (Mol) Stuntz) flour as protein and dietary fiber source in cookies and fried chips manufacture. Arch Latinoam Nutr. 2009; 59: 191-198.

18. Vidal A, Zaucedo A, Ramos M. Nutrimental properties of the sweet potato (Ipomoea batatas L.) and its benefits in human health. Rev Iber Tecnología Postcosecha. 2018; 19(2). 